\title{
Different subcellular localizations and functions of human ARD1 variants
}

\author{
JI HAE SEO ${ }^{1}$, JI-HYEON PARK ${ }^{1}$, EUN JI LEE ${ }^{1}$ and KYU-WON KIM ${ }^{1,2}$ \\ ${ }^{1}$ SNU-Harvard NeuroVascular Protection Research Center, College of Pharmacy and Research Institute \\ of Pharmaceutical Sciences, ${ }^{2}$ Department of Molecular Medicine and Biopharmaceutical Sciences, \\ Graduate School of Convergence Science and Technology, and College of Medicine or \\ College of Pharmacy, Seoul National University, Seoul 151-742, Republic of Korea
}

Received June 13, 2014; Accepted August 11, 2014

DOI: $10.3892 /$ ijo.2014.2770

\begin{abstract}
ARD1 is present in various species and has several variants derived from alternative splicing of mRNA. Previously, we reported differential biological functions and cellular distributions of mouse ARD1 (mARD1) variants. However, in comparison to mARD1 variants, human ARD1 (hARD1) variants have been rarely studied. In this study, we characterized a hARD1 variant, hARD ${ }^{131}$ and investigated its cellular activities. hARD ${ }^{131}$ mRNA was isolated from HeLa cells and sequenced. Sequence alignment revealed that, compared to hARD1 ${ }^{235}$, the most common form of hARD1, the mRNA sequence encoding hARD ${ }^{131}$ possesses an altered reading frame due to a 46-bp deletion. Thus, hARD $1^{131}$ and hARD1 ${ }^{235}$ differ in their C-terminal regions with a partially deleted acetyltransferase domain at the C-terminus of hARD1 ${ }^{131}$. Moreover, hARD $1^{131}$ and hARD $1^{235}$ showed different subcellular localizations and biological functions. hARD $1^{131}$ was mostly localized in the cell nucleus, whereas hARD ${ }^{235}$ was primarily localized in the cytoplasm. In addition, hARD ${ }^{235}$ stimulated cell proliferation by upregulation of cyclin D1, however hARD $1^{131}$ had no influence on cyclin D1 expression and cell growth. Because hARD ${ }^{235}$ enhances cell proliferation by its autoacetylation activity, we examined the autoacetylation activity of hARD1 ${ }^{131}$ and observed that this function was absent in hARD ${ }^{131}$. These results suggest that human ARD1 variants have different effects on cell proliferation, which may result from distinct subcellular localizations and autoacetylation activities.
\end{abstract}

\section{Introduction}

ARD1 was originally identified in yeast as an $\mathrm{N}$-acetyltransferase that catalyzes $\mathrm{N}$-terminal acetylation of newly

Correspondence to: Professor Kyu-Won Kim, SNU-Harvard NeuroVascular Protection Research Center, College of Pharmacy and Research Institute of Pharmaceutical Sciences, Seoul National University, Seoul 151-742, Republic of Korea

E-mail: qwonkim@snu.ac.kr

Key words: ARD1, splice variant, proliferation, cancer cells synthesized proteins. In yeast, ARD1 is required for entry into the stationary phase and sporulation during nitrogen deprivation (1). Subsequently, mammalian ARD1 was identified and found to catalyze not only N-terminal acetylation but also lysine acetylation of several proteins including hypoxiainducible factor-1 $\alpha$ (HIF-1 $\alpha$ ), $\beta$-catenin, myosin light chain kinase, the androgen receptor, tuberous sclerosis 2 (TSC2) and the tubulin complex (2-7). In mammalian cells, ARD1 regulates diverse cellular activities including growth, apoptosis, autophagy and differentiation (7-12). In particular, ARD1 garnered attention as a molecule that plays a critical role in cancer progression (13-15). ARD1 expression is elevated in various human cancers such as lung, breast, prostate, thyroid, and colorectal cancer (16-20). Furthermore, depletion of ARD1 leads to impaired proliferation or induces apoptosis in human cancer cells $(3,21)$. Thus, emerging evidence suggests that ARD1 could be a potential target for cancer therapy.

There are several isoforms of ARD1 derived from alternative splicing of mRNA. Alternative splicing of ARD1 mRNA is a species-specific event, thus isoform compositions differ between humans and mice (22). Previously, we identified three mouse (mARD1 ${ }^{198}, \mathrm{mARD}^{225}, \mathrm{mARD}^{235}$ ) and two human (hARD1 ${ }^{131}$, hARD1 ${ }^{235}$ ) ARD1 variants (23). Among these, $\mathrm{mARD}^{225}, \mathrm{mARD}^{235}$ and hARD ${ }^{235}$ have been well characterized and were found to have different cellular localizations with distinct roles in tumor angiogenesis $(2,22-27)$. However, the cellular expression profiles and biological functions of mARD ${ }^{198}$ and hARD1 ${ }^{131}$ remain unelucidated.

The current study was designed to characterize the hARD1 variant, hARD $1^{131}$ and to investigate how its cellular functions differ from hARD1 ${ }^{235}$, the most common form of hARD1. Our results demonstrate that the human ARD1 (hARD1) variants, hARD $1^{131}$ and hARD1 ${ }^{235}$, have different subcellular localizations and play distinct roles in the regulation of cell proliferation.

\section{Materials and methods}

Reagents and antibodies. Anti-GFP and cyclin D1 antibodies were purchased from Santa Cruz Biotechnology (Santa Cruz, CA, USA). Anti-acetyl-lysine antibody was purchased from Cell Signaling Technology (Danvers, MA, USA). Anti-tubulin was purchased from Sigma-Aldrich (St. Louis, MO, USA). 
Table I. Design of the primer sets for RT-PCR.

\begin{tabular}{llc}
\hline Primer & \multicolumn{1}{c}{ Sequence $\left(5^{\prime}-3^{\prime}\right)$} & Region (nucleotide) \\
\hline P1 sense & ATGAACATCCGCAATGCCAGG & $1-21$ \\
P1 antisense & CTAGGAGGCTGAGTCGGAGGC & $688-708$ \\
P2 sense & AACTTCAATGCCAAATATGTC & $301-321$ \\
P2 antisense & TCATGGCATAGGCGTCCTCCC & $422-442$ \\
P3 sense & AGCGGGACCTCACTCAGATGG & $443-463$ \\
P3 antisense & CTAGGAGGCTGAGTCGGAGGC & $688-708$ \\
\hline
\end{tabular}

The sequences of P1, P2 and P3 are conserved in hARD $1^{235}$ and hARD $1^{131}$ mRNA sequences. P1 sense and antisense primers correspond to the regions containing the start and stop codons of hARD $1^{235}$, respectively. P2 antisense primer corresponds to the region containing the stop codon of hARD $1^{131}$.

Cell culture. HeLa and $293 \mathrm{~T}$ cells were maintained in Dulbecco's modified Eagle's medium (DMEM) supplemented with $10 \%$ fetal bovine serum (FBS) at $37^{\circ} \mathrm{C}$ and $5 \% \mathrm{CO}_{2}$ in a humid atmosphere.

Plasmid constructions and transfection. To construct expression vectors for human ARD1 variants, ARD1 cDNA was amplified by PCR and sub-cloned into a GFP-tagged pCS2+ vector for cell expression, and a pGEX-4T vector for bacterial induction of the recombinant protein. Transfection was carried out using Lipofectamine (Life Technology, Carlsbad, CA, USA) or Polyfect (Qiagen, Valencia, CA, USA), according to the manufacturer's instructions.

Immunoblotting and immunoprecipitation. Cells were harvested and proteins were extracted using protein lysis buffer $(10 \mathrm{mM}$ HEPES at $\mathrm{pH} 7.9,40 \mathrm{mM} \mathrm{NaCl}, 0.1 \mathrm{mM}$ EDTA, 5\% glycerol, $1 \mathrm{mM}$ DTT and protease inhibitors). The concentration of extracted protein was measured using a BCA assay. Total cell lysates were resolved by SDS-PAGE and transferred onto nitrocellulose membranes (Amersham Pharmacia Bioscience, Piscataway, NJ, USA). The membrane was probed with a primary antibody followed by a secondary antibody conjugated to horseradish peroxidase, and protein was visualized using the ECL system (Intron Biotechnology, Gyeonggi-do, Korea).

In vitro acetylation assay. Recombinants of GST-hARD1 variants were freshly prepared as previously described (21). ARD1 recombinants were incubated in reaction mixture $(50 \mathrm{mM}$ Tris- $\mathrm{HCl}$ at $\mathrm{pH}$ 8.0, $0.1 \mathrm{mM}$ EDTA, $1 \mathrm{mM}$ DTT, $10 \%$ glycerol and $10 \mathrm{mM}$ acetyl-CoA) at $37^{\circ} \mathrm{C}$ for $1 \mathrm{~h}$.

Immunofluorescence staining and microscopy. Cells were placed on cover slips then incubated with Hoechst 33342 (Molecular Probes, Eugene, OR, USA) for nucleus staining. Axiovert M200 microscopes (Carl Zeiss, Jena, Germany) were used for immunofluorescence imaging.

Reverse transcription-PCR analysis. Total RNA was extracted using an RNA extraction kit (Invitrogen, Carlsbad, CA, USA). cDNA was synthesized from $2 \mu \mathrm{g}$ of RNA using an oligo(dt) primer. Primers used for PCR reactions were as follows: human ARD1, 5'-ATGAACATCCGCAATGCGAG-3' (forward) and 5'-CTCATATCATGGCTCGAGAGG-3' (reverse); cyclin D1, 5'-CTGGCCATGAACTACCTGGA-3' (forward) and 5'-GTC ACACTTGATCACTCTGG-3' (reverse); GAPDH, 5'-ACCAC AGTCCATGCCATCAC-3' (forward) and 5'-TCCACCACCCT GTTGCTGTA-3' (reverse). The PCR reaction was performed for 25 cycles to allow ARD1, cyclin D1 and GAPDH amplification.

Cell proliferation assay. The rate of cell proliferation was measured using a Non-Radioactive Proliferation Assay kit (Promega) according to the manufacturer's instructions. Briefly, cells were seeded into 96-well plates and cultured for three days. Subsequently, $20 \mu 1$ of substrate solution was added and the cells were incubated for $1 \mathrm{~h}$ to allow color development. The absorbance at $492 \mathrm{~nm}$ was measured to determine the number of proliferating cells.

Statistical analysis. Results are presented as means \pm SD and P-values were calculated by applying the two-tailed Student's $\mathrm{t}$-test to data derived from three independent experiments. Differences were considered statistically significant when $\mathrm{P}<0.05$.

\section{Results}

Expression of hARDl variants. During the cloning of human ARD1 using mRNA prepared from HeLa cells, we observed that cDNA from one clone (no. 4) was shorter than cDNA from other clones (Fig. 1A). Sequence analysis revealed that the clone with the shorter cDNA sequence (clone no. 4) was hARD1 ${ }^{131}$ (GenBank accession no. BC063377), whereas the other clones were all hARD1 ${ }^{235}$ (GenBank accession no. NM_003491). As shown in Fig. 1B, the nucleotide sequence from residue 342 to 387, which is located in exon 6 and exon 7 of ARD1 mRNA, was deleted in hARD $1^{131}$. Therefore, the nucleotide sequence of hARD1 ${ }^{131}$ is 46 bp shorter than that of hARD1 ${ }^{235}$.

To confirm the expression of hARD $1^{131}$ in human cells, we performed RT-PCR using mRNA isolated from 293T and HeLa cells. As described in Table I and Fig. 1B, three kinds of primer sets named $\mathrm{P} 1, \mathrm{P} 2$ and $\mathrm{P} 3$ were designed for the PCR analysis: P1 and P2 contained the deleted mRNA 
A

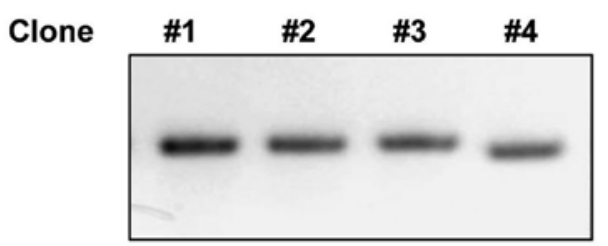

B

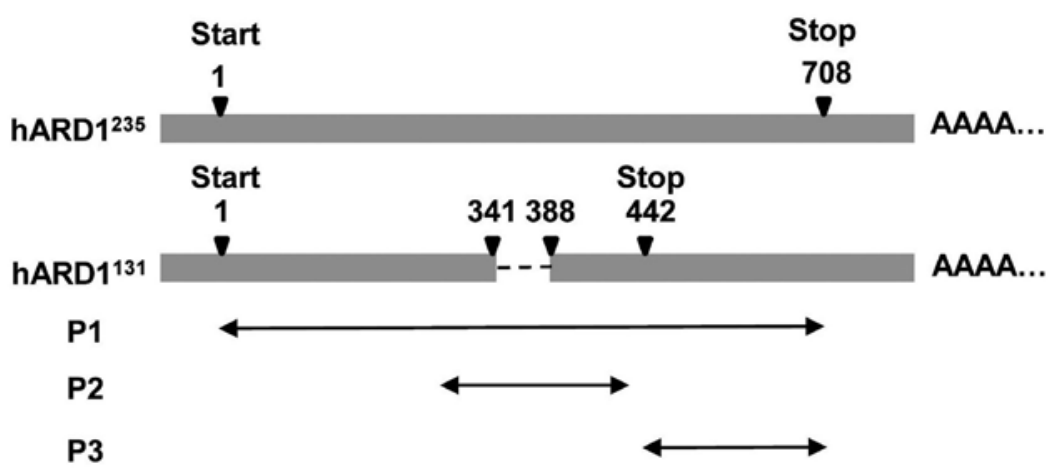

C

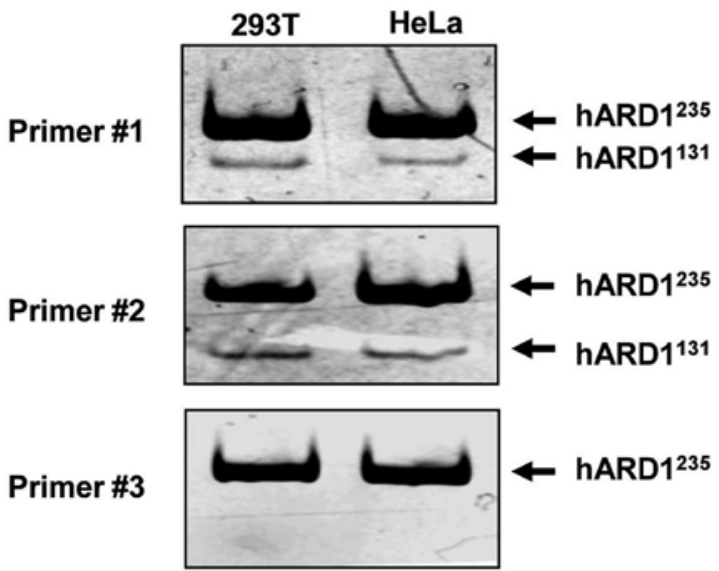

Figure 1. Expression of hARD1 variants in human cell lines. (A) hARD1 sequence was amplified from cDNA reverse transcribed from HeLa cell mRNA. Four clones were analyzed by electrophoresis on agarose gels. (B) cDNA alignment of hARD1 ${ }^{235}$ and hARD1 ${ }^{131}$. Primers sets P1, P2 and P3 were designed for the detection of hARD1 ${ }^{235}$ and hARD1 ${ }^{131}$. (C) Total RNA was exacted from 293T and HeLa cells and RT-PCR was performed using primer sets P1, P2 and P3. Expression of hARD1 ${ }^{235}$ and hARD $1^{131}$ was analyzed by electrophoresis on DNA polyacrylamide gels.

region, whereas $\mathrm{P} 3$ did not. As predicted, when $\mathrm{P} 1$ and $\mathrm{P} 2$ were used in PCR, two bands corresponding to hARD1 ${ }^{131}$ and hARD1 ${ }^{235}$ were detected by polyacrylamide gel electrophoresis. However, only one band, corresponding to hARD $1^{235}$, was detected following PCR with the P3 primer set. These data confirm the expression of the hARD $1^{131}$ splice variant in human cells (Fig. 1C).

Sequence comparison of hARDl variants. Next, we compared the coding sequences of hARD $1^{131}$ and hARD1 ${ }^{235}$. The coding sequence of hARD1 ${ }^{235}$ terminates at amino acid 708 , thus it encodes a 235 amino acid proteins. However, in hARD $1^{131}$, deletion of $46 \mathrm{bp}$ in hARD1 ${ }^{131}$ results in a frame shift after amino acid 113 , resulting in premature termination at amino acid 131 (Fig. 2A and B). ARD1 is predicted to have an acetyltransferase domain located between amino acid residues 45 and 130, in which an acetyl-CoA binding domain is positioned between amino acid residues 82 and 87 . Compared with hARD $1^{235}$, hARD $1^{131}$ protein possesses a conserved acetylCoA binding domain. However, $20 \%$ of the acetyltransferase domain was deleted in $\mathrm{hARD}^{131}$ and it was found to have a different C-terminal region (Fig. 2B).

Subcellular localization of hARDl variants. In a previous study, we reported that splice variants of mouse ARD1 have a different subcellular localization (22). Therefore, we speculated that hARD $1^{131}$ might have a distinct localization compared to hARD1 ${ }^{235}$. To investigate ARD1 location in human cells, we constructed GFP-tagged plasmids containing hARD $1^{131}$ and hARD $1^{235}$, which were subsequently transfected into HeLa and 293T cells. The localization of GFP-hARD1 variants and control GFP protein was analyzed by fluorescence microscopy. As shown in Fig. 3, GFP-hARD1 ${ }^{235}$ was observed predominantly in the cytoplasm, despite hARD1 containing a putative nuclear localization sequence (NLS) (Fig. 2B). however, hARD1 ${ }^{131}$ was specifically located in the cell nucleus. These results demonstrate that hARD1 variants have distinct subcellular localizations, and suggest that the roles of hARD $1^{131}$ and hARD $1^{235}$ might differ within the cell. 
h235 ATGAACATCCGCAATGCGAGGCCAGAGGACCTAATGAACATGCAGCACTGCAACCTCCTC 60 h131 ATGAACATCCGCAATGCGAGGCCAGAGGACCTAATGAACATGCAGCACTGCAACCTCCTC 60

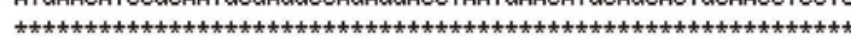

\section{B}

\section{hARD1235}

TGCCTGCCCGAGAACTACCAGATGAAATACTACTTCTACCATGGCCTTTCCTGGCCCCAG 120 TGCCTGCCCGAGAACTACCAGATGAAATACTACTTCTACCATGGCCTTTCCTGGCCCCAG 120

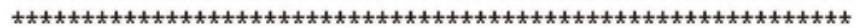

СTCTCTTACATTGCTGAGGACGÁGÄATGGGAAGATTGTGGGGTATGTCCTGGCCAAÁATG 180 CTCTCTTACATTGCTGAGGACGAGAATGGGAAGATTGTGGGGTATGTCCTGGCCAAAATG 180

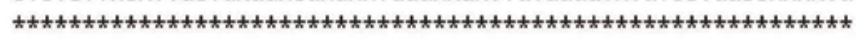

GAAGAGGACCCAGATGATGTGCCCCATGGACATATCACCTCATTGGCTGTGAAGCGTTCC 240 GAAGAGGACCCAGATGATGTGCCCCATGGACATATCACCTCATTGGCTGTGAAGCGTTCC 240

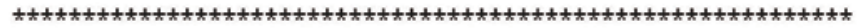

CACCGGCGCCTCGGTCTGGCTCAGAAACTGATGGACCAGGCCTCTCGAGCCATGATAGAG 300 CACCGGCGCCTCGGTCTGGCTCAGAAACTGATGGACCAGGCCTCTCGAGCCATGATAGAG 300

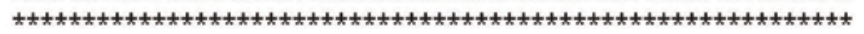

AACTTCAATGCCAAATATGTCTCCCTGCATGTCAGGAAGAGTAACCGGGCCGCCCTGCAC 360 AACTTCAATGCCAAATATGTCTCCCTGCATGTCAGGAAGAG----- 341

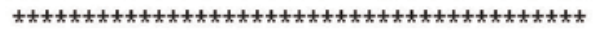

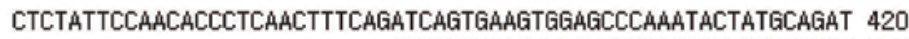
-ATCAGTGAAGTGGAGCCCAAATACTATGCAGAT 374

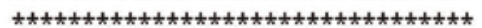

GGGGAGGACGCCTATGCCATGAAGCGGGACCTCACTCAGATGGCCGACGAGCTGAGGCGG 480

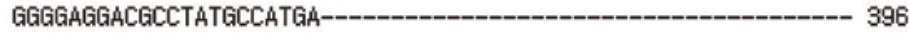
**********************

CACCTGGAGCTGAAAGAGAAGGGCAGGCACGTGGTGCTGGGTGCCATCGAGAACAAGGTG 540

GAGAGCAAAGGCAATTCACCTCCGAGCTCAGGAGAGGCCTGTCGCGAGGAGAAGGGCCTG 600

GCTGCCGAGGATAGTGGTGGGGACAGCAAGGACCTCAGCGAGGTCAGCGAGACCACAGAG 660

\section{AGCACAGATGTCAAGGACAGCTCAGAGGCCTCCGACTCAGCCTCCTAG 708}
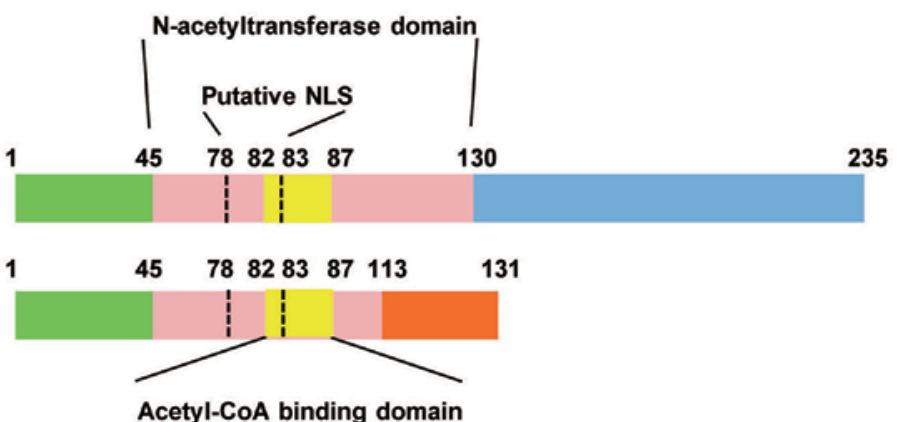

Figure 2. Sequence comparison of human ARD1 (hARD1) variants. (A) Sequence alignment of hARD1 $1^{235}$ and hARD1 ${ }^{131}$. Identical residues are indicated by asterisk. (B) Schematic representation of hARD1 ${ }^{235}$ and hARD1 ${ }^{131}$ amino acid sequences. The acetyltransferase domain at amino acid $45-130$, the putative NLS at amino acid 78-83 and the acetyl-CoA binding domain at amino acid 82-87 are indicated.

Different functions of hARDl variants in the regulation of cell proliferation. Several studies have reported that hARD $1^{235}$ regulates the cell cycle and stimulates cell proliferation $(3,21)$. Thus, we aimed to determine whether hARD1 ${ }^{131}$ could promote cellular growth in a similar way to hARD1 ${ }^{235}$. Consistent with previous studies, compared to control cells, cell proliferation was significantly accelerated in hARD $1^{235}$ expressing HeLa cells. However, hARD $1^{131}$ had no effect on cell growth, suggesting that hARD1 variants have different roles in the regulation of cell proliferation (Fig. 4A and B). In a previous study, cyclin D1 was found to mediate ARD1-induced cell growth, therefore we examined the expression levels of cyclin D1 mRNA and protein in hARD1 $1^{235}$ and hARD1 ${ }^{235}$ transfected cells $(3,21)$. Consistent with enhanced cellular growth, cyclin D1 mRNA and protein expression were significantly increased in hARD1 ${ }^{235}$, but not hARD $1^{131}$ transfected cells (Fig. 4C and D). These results suggest that the diverse roles of hARD1 variants in the regulation of cell proliferation may be due to their different effects on cyclin D1 expression.

Previously, we showed that the autoacetylation activity of hARD $1^{235}$ was required for enhanced cell proliferation (21). Thus, we compared the autoacetylation activities of hARD1 $1^{131}$ 
A
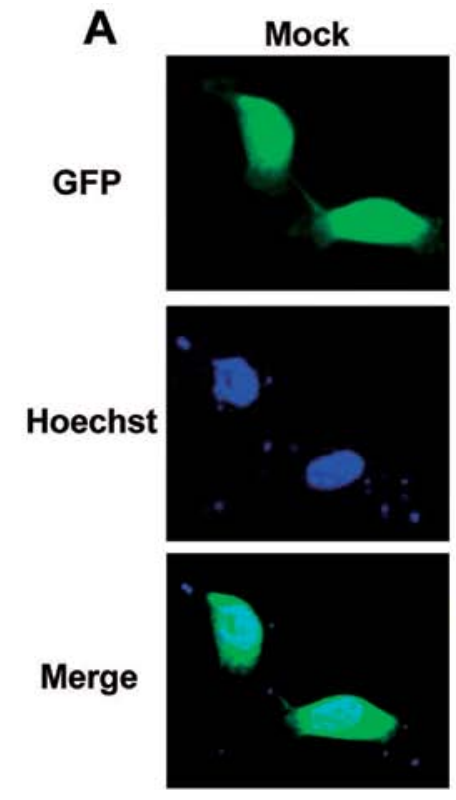

B
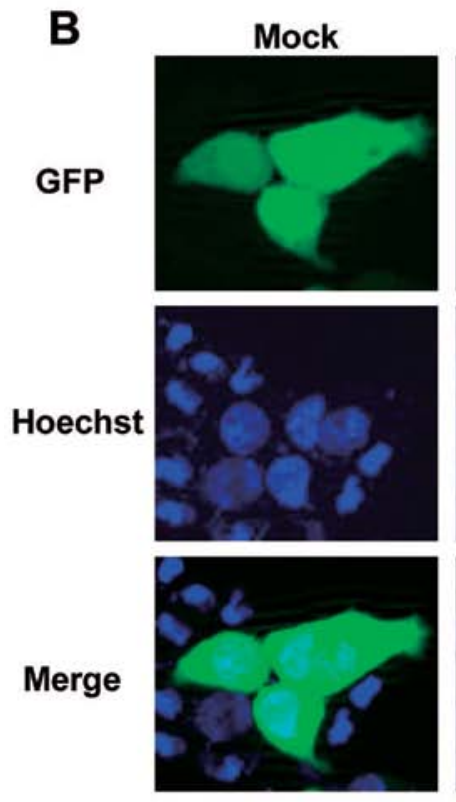

hARD1235
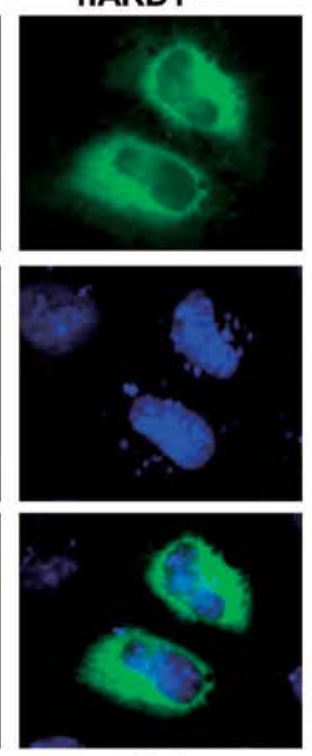

HeLa
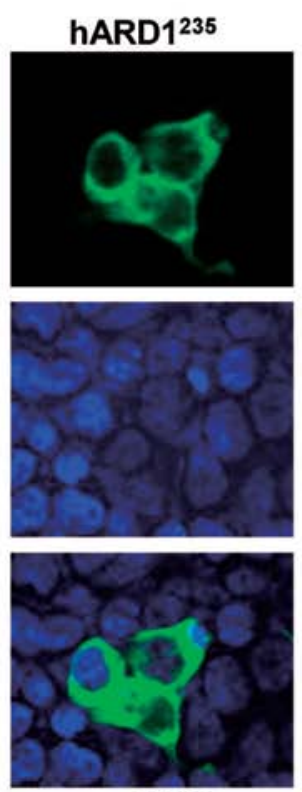

293T
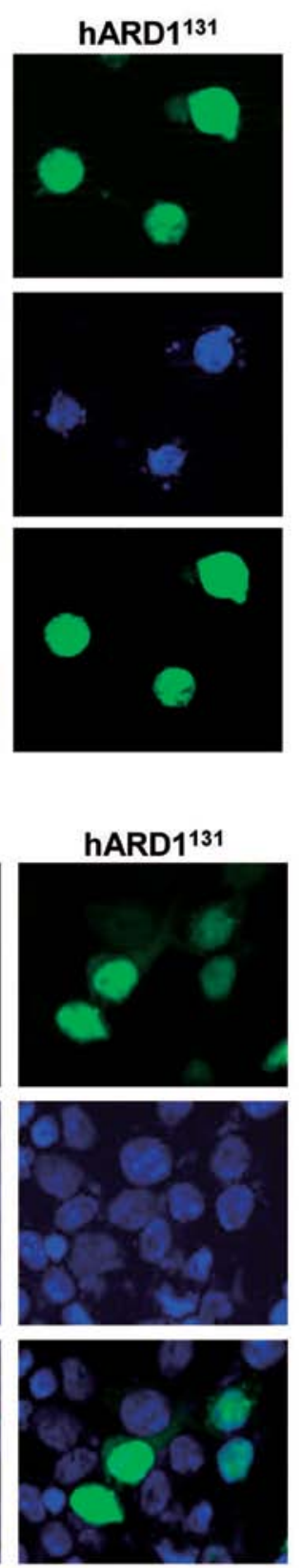

Figure 3. Subcellular localization of hARD1 variants in human cell lines. GFP-tagged plasmids for hARD1 variants were transfected into HeLa cells (A) and 293 T cells (B). Nucleus-staining was performed with Hoechst. Localization of ARD1 variants was analyzed and photographed by a fluorescence microscope.

and hARD1 $1^{235}$ using an in vitro acetylation assay. While the hARD1 ${ }^{235}$ recombinant acetylated itself, hARD1 ${ }^{131}$ was not acetylated in vitro, indicating a lack of autoacetylation activity in this splice variant (Fig. 4E). These results suggest that, unlike hARD $1^{235}$, hARD $1^{131}$ has no autoacetylation activity, and is therefore unable to upregulate cyclin D1 expression and promote cell proliferation.

\section{Discussion}

Alternative mRNA splicing is a common process in the regulation of gene expression by which a single gene codes for multiple proteins. This process contributes to protein diversity, and different proteins produced from alternative splicing often have distinct cellular functions $(28,29)$. The current study characterized alternative splice variants of human ARD1 and demonstrated differential biological functions and cellular distributions of these variants.

Previously, we identified two hARD1 variants, hARD1 $1^{235}$ and hARD $1^{131}$ (23). However, human cells dominantly express hARD1 ${ }^{235}$, and hARD1 ${ }^{131}$ expression has not been detected using RT-PCR or western blots in previous studies $(22,23)$. In this study, the expression of hARD $1^{131}$ was clearly detected using RT-PCR and polyacrylamide gel electrophoresis, which can separate DNA well beyond the resolving capabilities of an agarose gel (Fig. 1C). The basal expression level of hARD1 ${ }^{131}$ was relatively lower than that of hARD1 ${ }^{235}$. Therefore, we could not exclude the possibility that the cellular activity 

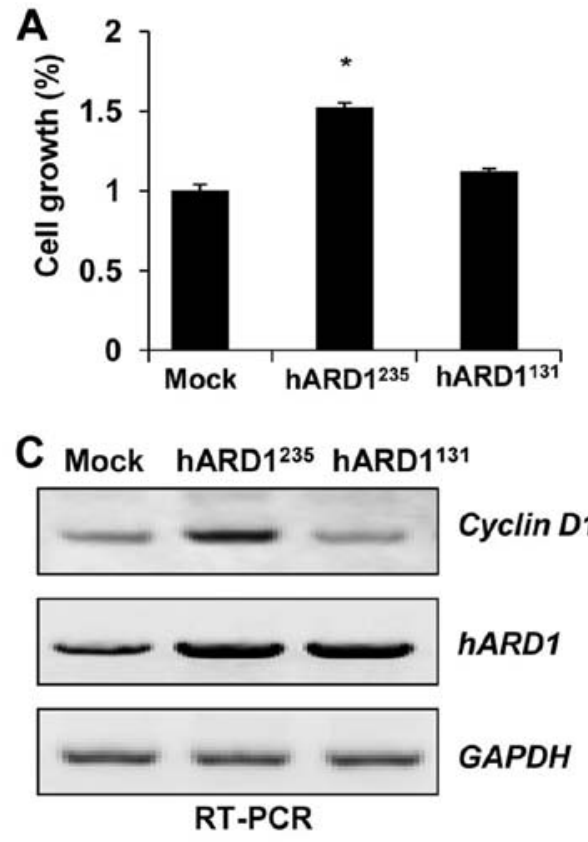

E

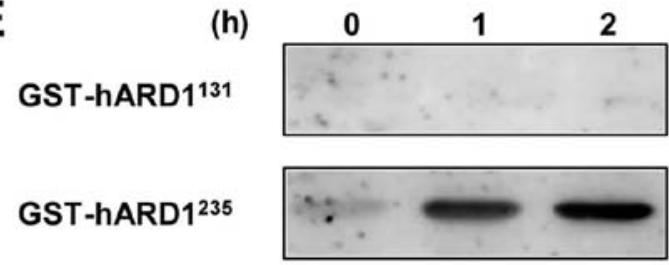

WB : Anti-Lys-Ac

B

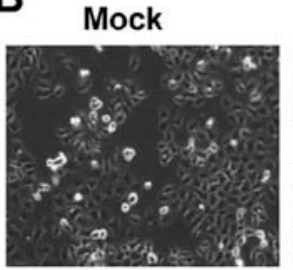

hARD1235

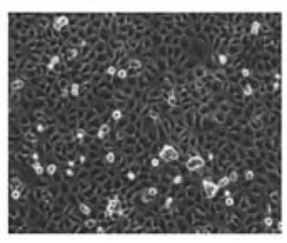

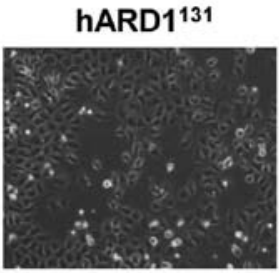

D

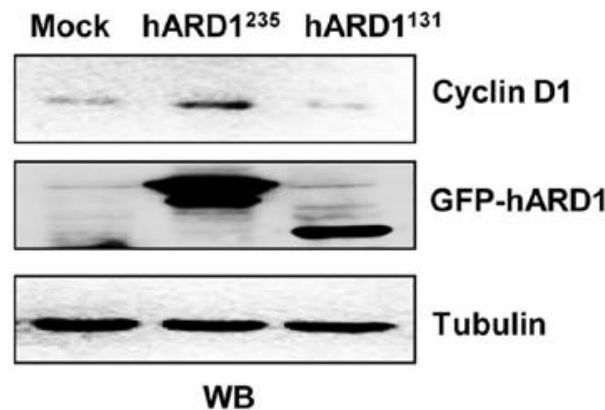

(h)
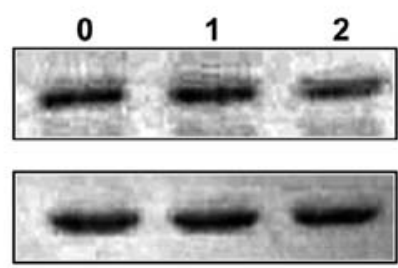

Input

Figure 4. Differential functions of human ARD1 (hARD1) variants in the regulation of cell proliferation. (A) HeLa cells were transfected with GFP-tagged hARD1 $1^{235}$ and hARD1 ${ }^{131}$, then cell growth was analyzed ${ }^{*} \mathrm{P}<0.05$ vs. control. (B) After transfecting cells with plasmids containing hARD1 $1^{235}$ and hARD1 ${ }^{131}$, cells were grown for 3 days and photographed. (C, D) GFP-tagged hARD1 ${ }^{235}$ and hARD1 ${ }^{131}$ plasmids were transfected into HeLa cells. Cyclin D1 mRNA and protein levels were analyzed by RT-PCR and western blot, respectively. (E) Purified recombinants for GST-hARD1 ${ }^{235}$ and GST-hARD1 ${ }^{131}$ were each subjected to the in vitro acetylation assay for 1 and $2 \mathrm{~h}$. Acetylated proteins were detected using an anti-acetyl lysine (Lys-Ac) antibody. Total proteins were stained with Coomassie Brilliant Blue (Input).

of hARD $1^{131}$ might be small or performed preferentially by hARD1 $1^{235}$

Interestingly, hARD $1^{131}$ has a unique subcellular localization compared to hARD1235 (Fig. 3). Although the NLS is conserved at amino acid residues 78-83 in all ARD1 variants, the different subcellular localizations of hARD1 variants could be explained by structural difference in C-terminal regions (Fig. 2).

The subcellular localization of a protein corresponds to its biological function. As an $\mathrm{N}$-acetyltransferase, hARD1 ${ }^{235}$ cooperates with NATH-1 for N-terminal acetylation of newly synthesized proteins in the cytoplasm (14). However, the nuclear localization of hARD1 ${ }^{131}$ suggests that it might have functions other than $\mathrm{N}$-acetylation. Indeed, $\mathrm{N}$-terminal acetyltransferase activity is associated with cellular growth. However, hARD1 ${ }^{131}$ had no effect on cellular growth (Fig. 4A and B). Moreover, autoacetylation activity, which is essential for the ability of hARD1 ${ }^{235}$ to stimulate cell proliferation, was also absent in the hARD $1^{131}$ variant (Fig. 4E). These results suggest novel functions of hARD ${ }^{131}$ that differ from that of hARD $1^{235}$, and suggest the necessity for further experiments to investigate the diverse cellular functions of hARD1 ${ }^{131}$.
In summary, the present study revealed that hARD1 variants have different cell proliferative activities that might be associated with their different subcellular localizations and enzymatic activities. To further our understanding of hARD1 isoforms, future studies will focus on elucidating the specific roles played by hARD1 variants and their relationships under various physiological conditions.

\section{Acknowledgements}

This research was supported by the Global Research Laboratory Program (2011-0021874), Global Core Research Center (GCRC) Program (2011-0030001), National Research Foundation (NRF) grant (2013-036038) funded by the Ministry of Science, ICT and Future Planning (MSIP) and Basic Science Research Program through the NRF of Korea funded by the Ministry of Education (2013R1A1A2058956).

\section{References}

1. Driessen HP, de Jong WW, Tesser GI and Bloemendal H: The mechanism of N-terminal acetylation of proteins. CRC Crit Rev Biochem 18: 281-325, 1985. 
2. Jeong JW, Bae MK, Ahn MY, et al: Regulation and destabilization of HIF-1alpha by ARD1-mediated acetylation. Cell 111: 709-720, 2002.

3. Lim JH, Park JW and Chun YS: Human arrest defective 1 acetylates and activates beta-catenin, promoting lung cancer cell proliferation. Cancer Res 66: 10677-10682, 2006.

4. Ohkawa N, Sugisaki S, Tokunaga E, et al: N-acetyltransferase ARD1-NAT1 regulates neuronal dendritic development. Genes Cells 13: 1171-1183, 2008

5. Shin DH, Chun YS, Lee KH, Shin HW and Park JW: Arrest defective-1 controls tumor cell behavior by acetylating myosin light chain kinase. PLoS One 4: e7451, 2009.

6. Wang Z, Wang Z, Guo J, et al: Inactivation of androgen-induced regulator ARD1 inhibits androgen receptor acetylation and prostate tumorigenesis. Proc Natl Acad Sci USA 109: 3053-3058, 2012.

7. Kuo HP, Lee DF, Chen CT, et al: ARD1 stabilization of TSC2 suppresses tumorigenesis through the mTOR signaling pathway. Sci Signal 3: ra9, 2010.

8. Xu H, Jiang B, Meng L, et al: $\mathrm{N}-\alpha$-acetyltransferase 10 protein inhibits apoptosis through RelA/p65-regulated MCL1 expression. Carcinogenesis 33: 1193-1202, 2012.

9. Yi CH, Sogah DK, Boyce M, Degterev A, Christofferson DE and Yuan J: A genome-wide RNAi screen reveals multiple regulators of caspase activation. J Cell Biol 179: 619-626, 2007.

10. Arnesen T, Gromyko D, Pendino F, Ryningen A, Varhaug JE and Lillehaug JR: Induction of apoptosis in human cells by RNAi-mediated knockdown of hARD1 and NATH, components of the protein $\mathrm{N}$-alpha-acetyltransferase complex. Oncogene 25 : 4350-4360, 2006.

11. Asaumi M, Iijima K, Sumioka A, et al: Interaction of N-terminal acetyltransferase with the cytoplasmic domain of beta-amyloid precursor protein and its effect on A beta secretion. J Biochem 137: 147-155, 2005.

12. Arnesen T, Anderson D, Baldersheim C, Lanotte M, Varhaug JE and Lillehaug JR: Identification and characterization of the human ARD1-NATH protein acetyltransferase complex Biochem J 386: 433-443, 2005.

13. Kuo HP and Hung MC: Arrest-defective-1 protein (ARD1): tumor suppressor or oncoprotein? Am J Transl Res 2: 56-64, 2010.

14. Kalvik TV and Arnesen T: Protein N-terminal acetyltransferases in cancer. Oncogene 32: 269-276, 2013.

15. Arnesen T, Thompson PR, Varhaug JE and Lillehaug JR: The protein acetyltransferase ARD1: a novel cancer drug target? Curr Cancer Drug Targets 8: 545-553, 2008.
16. Shim JH, Chung YH, Kim JA, et al: Clinical implications of arrest-defective protein 1 expression in hepatocellular carcinoma: a novel predictor of microvascular invasion. Dig Dis 30: 603-608, 2012.

17. Wang ZH, Gong JL, Yu M, et al: Up-regulation of human arrestdefective 1 protein is correlated with metastatic phenotype and poor prognosis in breast cancer. Asian Pac J Cancer Prev 12: 1973-1977, 2011.

18. Yu M, Gong J, Ma M, et al: Immunohistochemical analysis of human arrest-defective-1 expressed in cancers in vivo. Oncol Rep 21: 909-915, 2009.

19. Ren T, Jiang B, Jin G, et al: Generation of novel monoclonal antibodies and their application for detecting ARD1 expression in colorectal cancer. Cancer Lett 264: 83-92, 2008.

20. Arnesen T, Gromyko D, Horvli O, Fluge O, Lillehaug J and Varhaug JE: Expression of $\mathrm{N}$-acetyl transferase human and human arrest defective 1 proteins in thyroid neoplasms. Thyroid 15: 1131-1136, 2005.

21. Seo JH, Cha JH, Park JH, et al: Arrest defective 1 autoacetylation is a critical step in its ability to stimulate cancer cell proliferation. Cancer Res 70: 4422-4432, 2010.

22. Chun KH, Cho SJ, Choi JS, Kim SH, Kim KW and Lee SK: Differential regulation of splicing, localization and stability of mammalian ARD1235 and ARD1225 isoforms. Biochem Biophys Res Commun 353: 18-25, 2007.

23. Kim SH, Park JA, Kim JH, et al: Characterization of ARD1 variants in mammalian cells. Biochem Biophys Res Commun 340: 422-427, 2006

24. Arnesen T, Kong X, Evjenth R, et al: Interaction between HIF-1 alpha (ODD) and hARD1 does not induce acetylation and destabilization of HIF-1 alpha. FEBS Lett 579: 6428-6432, 2005.

25. Bilton R, Mazure N, Trottier E, et al: Arrest-defective-1 protein, an acetyltransferase, does not alter stability of hypoxia-inducible factor (HIF)-1alpha and is not induced by hypoxia or HIF. J Biol Chem 280: 31132-31140, 2005.

26. Fisher TS, Etages SD, Hayes L, Crimin K and Li B: Analysis of ARD1 function in hypoxia response using retroviral RNA interference. J Biol Chem 280: 17749-17757, 2005.

27. Lee MN, Lee SN, Kim SH, et al: Roles of arrest-defective protein $1(225)$ and hypoxia-inducible factor 1alpha in tumor growth and metastasis. J Natl Cancer Inst 102: 426-442, 2010.

28. Brett D, Pospisil H, Valcarcel J, Reich J and Bork P: Alternative splicing and genome complexity. Nat Genet 30: 29-30, 2002.

29. Nilsen TW and Graveley BR: Expansion of the eukaryotic proteome by alternative splicing. Nature 463: 457-463, 2010. 